Mathematical Sciences And Applications E-Notes

Volume 2 No. 1 PP. 99-105 (2014) @ MSAEN

\title{
HYPERSURFACES SATISFYING SOME CURVATURE CONDITIONS ON PSEUDO PROJECTIVE CURVATURE TENSOR IN THE SEMI-EUCLIDEAN SPACE
}

\author{
YUSUF DOĞRU
}

(Communicated by Maria FALCITELLI)

\begin{abstract}
We consider some curvature conditions on the Pseudo projective curvature tensor $\widetilde{P}$ on a hypersurface in the semi-Euclidean space $E_{s}^{n+1}$. We prove that every pseudo projectively Ricci-semisymmetric hypersurface $M$ satisfying the condition $\widetilde{P} \cdot R=0$ is pseudosymmetric. We also consider the condition $\widetilde{P} \cdot S=0$ on hypersurfaces of the semi-Euclidean space $E_{s}^{n+1}$.
\end{abstract}

\section{Introduction}

Let $(M, g)$ be an $n$-dimensional, $n \geq 3$, differentiable manifold of class $C^{\infty}$. The pseudo projective curvature tensor $\widetilde{P}$ was introduced by B.Prasad [11]. According to them, a pseudo projective curvature tensor is defined by

$$
\begin{aligned}
\widetilde{P}(X, Y) Z= & a R(X, Y) Z+b[S(Y, Z) X-S(X, Z) Y] \\
& -\frac{\kappa}{n}\left[\frac{a}{n-1}+b\right](g(Y, Z) X-g(X, Z) Y),
\end{aligned}
$$

where $a$ and $b$ are constants, $S$ is the Ricci tensor and $\kappa$ is the scalar curvature of the manifold $M$.

In [7], Dabrowska, Defever, Deszcz and Kowalczyk studied semisymmetry and Ricci-semisymmetry for hypersurfaces of semi-Euclidean space. Recently in [8], Özgür studied hypersurfaces satisfying some curvature conditions in the semiEuclidean space. In [10], Özgür, Arslan and Murathan studied conharmonically semiparallel hypersurfaces in Euclidean space. In [9], Özgür and Arslan studied pseudosymmetric hypersurfaces satisfying Chen's equality in Euclidean space. In the present study, our aim is to study hypersurfaces of dimension $n \geq 4$, in $(n+1)$ dimensional semi-Euclidean space $E_{s}^{n+1}$. We show that if a pseudo projectively Ricci-semisymmetric hypersurface $M$ satisfies the condition $\widetilde{P} \cdot R=0$, where $R$ denotes the curvature tensor of $M$, then $M$ is pseudosymmetric.

Date: Received: October 2, 2013; Revised: January 20, 2014; Accepted: February 18, 2014. 2010 Mathematics Subject Classification. 53B20, 53C25, 53B30, 53C40.

Key words and phrases. Pseudo projective curvature tensor, pseudosymmetric manifold, Ricci-semisymmetric manifold. 
The paper is organized as follows: In Section 2, we give a brief account of pseudo projective curvature tensor, pseudosymmetric manifolds and Kulkarni-Nomizu product. In Section 3, we give some information about hypersurfaces of semi-Euclidean space $E_{s}^{n+1}$ and the main results of the study are presented.

\section{Preliminaries}

We denote by $\nabla, R, \widetilde{P}, S$ and $\kappa$ are the Levi-Civita connection, the RiemannianChristoffel curvature tensor, the pseudo projective curvature tensor, the Ricci tensor and the scalar curvature of $(M, g)$, respectively. Next, we define the endomorphisms $\mathcal{R}(X, Y)$ and $\widetilde{P}(X, Y)$ of $\chi(M)$ by

and

$$
\mathcal{R}(X, Y) Z=\left[\nabla_{X}, \nabla_{Y}\right] Z-\nabla_{[X, Y]} Z
$$

$$
\widetilde{P}(X, Y) Z=a \mathcal{R}(X, Y) Z+b\left(X \Lambda_{S} Y\right) Z-\frac{\kappa}{n}\left[\frac{a}{n-1}+b\right](X \Lambda Y) Z,
$$

respectively, where $(X \Lambda Y) Z$ is the tensor, defined by

$$
(X \Lambda Y) Z=g(Y, Z) X-g(X, Z) Y
$$

and $X, Y, Z \in \chi(M)$.

The Riemannian-Christoffel curvature tensor $R$ and the pseudo projective curvature tensor $\widetilde{P}$ are defined by

$$
\begin{aligned}
& R(X, Y, Z, W)=g(\mathcal{R}(X, Y) Z, W), \\
& \widetilde{P}(X, Y, Z, W)=g(\widetilde{\mathcal{P}}(X, Y) Z, W),
\end{aligned}
$$

respectively, where $W \in \chi(M)$. The $(0,4)$-tensor $G$ is defined by $G(X, Y, Z, W)=$ $g((X \wedge Y) Z, W)$.

For a $(0, k)$-tensor field $T, k \geq 1$, and a $(0,2)$-tensor field $E$ on $(M, g)$ we define the tensors $R \cdot T, \widetilde{P} \cdot T$, and $Q(E, T)$ by

$$
\begin{aligned}
(R(X, Y) \cdot T)\left(X_{1}, \ldots, X_{k}\right)= & -T\left(\mathcal{R}(X, Y) X_{1}, X_{2}, \ldots, X_{k}\right) \\
& -\ldots-T\left(X_{1}, \ldots, X_{k-1}, \mathcal{R}(X, Y) X_{k}\right) . \\
(\widetilde{P}(X, Y) \cdot T)\left(X_{1}, \ldots, X_{k}\right)=- & T\left(\widetilde{\mathcal{P}}(X, Y) X_{1}, X_{2}, \ldots, X_{k}\right) \\
& -\ldots-T\left(X_{1}, \ldots, X_{k-1}, \widetilde{\mathcal{P}}(X, Y) X_{k}\right) . \\
Q(E, T)\left(X_{1}, \ldots, X_{k} ; X, Y\right)=- & T\left(\left(X \wedge_{E} Y\right) X_{1}, X_{2}, \ldots, X_{k}\right) \\
& -\ldots-T\left(X_{1}, \ldots, X_{k-1},\left(X \wedge_{E} Y\right) X_{k}\right),
\end{aligned}
$$

respectively, where the tensor $X \wedge_{E} Y$ is defined by

$$
\left(X \wedge_{E} Y\right) Z=E(Y, Z) X-E(X, Z) Y .
$$

If $E=g$ then we simply denote it by $X \wedge Y$.

If the tensor $R \cdot R$ and $Q(g, R)$ are linearly dependent, then $M$ is called pseudosymmetric. This is equivalent to

$$
R \cdot R=L_{R} Q(g, R)
$$

holding on the set $U_{R}=\left\{x \in M^{n} \mid Q(g, R) \neq 0\right.$ at $\left.x\right\}$, where $L_{R}$ is some function on $U_{R}$ (see[5]). If $R \cdot R=0$, then $M$ is called semi-symmetric (see[12]). 
If the tensors $R \cdot S$ and $Q(g, S)$ are linearly dependent, then $M$ is called Riccipseudosymmetric. This is equivalent to

$$
R \cdot S=L_{S} Q(g, S)
$$

holding on the set $U_{S}=\left\{x \in M^{n} \mid S-\frac{\kappa}{n} g \neq 0\right.$ at $\left.x\right\}$, where $L_{S}$ is some function on $U_{S}($ see $[5])$.

The Kulkarni-Nomizu product $E \widetilde{\wedge} B$ is given by

$$
\begin{aligned}
(E \widetilde{\wedge} B)\left(X_{1}, X_{2}, X_{3}, X_{4}\right)= & E\left(X_{1}, X_{4}\right) B\left(X_{2}, X_{3}\right)+E\left(X_{2}, X_{3}\right) B\left(X_{1}, X_{4}\right) \\
& -E\left(X_{1}, X_{3}\right) B\left(X_{2}, X_{4}\right)-E\left(X_{2}, X_{4}\right) B\left(X_{1}, X_{3}\right) .
\end{aligned}
$$

We note that if $E=B$, then we have $\bar{E}=\frac{1}{2} E \widetilde{\wedge} E$, where the (0,4)-tensor $\bar{E}$ is defined by

$$
\bar{E}\left(X_{1}, X_{2}, X_{3}, X_{4}\right)=E\left(X_{1}, X_{4}\right) E\left(X_{2}, X_{3}\right)-E\left(X_{1}, X_{3}\right) E\left(X_{2}, X_{4}\right) .
$$

Further, for a symmetric $(0,2)$-tensor $E$ and a $(0, k)$-tensor $T, k \geq 2$, we define their Kulkarni-Nomizu product $E \widetilde{\wedge} T$ by

$$
\begin{aligned}
(E \widetilde{\wedge} T)\left(X_{1}, X_{2}, X_{3}, X_{4} ; Y_{3}, \ldots, Y_{k}\right)= & E\left(X_{1}, X_{4}\right) T\left(X_{2}, X_{3} ; Y_{3}, \ldots, Y_{k}\right) \\
& +E\left(X_{2}, X_{3}\right) T\left(X_{1}, X_{4} ; Y_{3}, \ldots, Y_{k}\right) \\
& -E\left(X_{1}, X_{3}\right) T\left(X_{2}, X_{4} ; Y_{3}, \ldots, Y_{k}\right) \\
& -E\left(X_{2}, X_{4}\right) T\left(X_{1}, X_{3} ; Y_{3}, \ldots, Y_{k}\right)
\end{aligned}
$$

(see [4]). For symmetric $(0,2)$-tensor field $E$ and $B$, we have the following identity $([4]):$

$$
E \widetilde{\wedge} Q(B, E)=Q(B, \bar{E})
$$

Note that

$$
\bar{g}=G
$$

\section{Hypersurfaces}

Let $M, n=\operatorname{dim} M \geq 3$, be a connected hypersurface immersed isometrically in a semi-Riemannian manifold $(N, \widetilde{g})$. We denote by $g$ the metric tensor of $M$ induced from the metric tensor $\widetilde{g}$. Further, we denote by $\tilde{\nabla}$ and $\nabla$ the Levi-Civita connections corresponding to the metric tensors $\widetilde{g}$ and $g$, respectively. Let $\xi$ be a local unit vector field on $M$ in $N$ and let $\varepsilon=\widetilde{g}(\xi, \xi)= \pm 1$. We can present the Gauss formula and Weingarten formula of $M$ in $N$ in the following form:

$$
\tilde{\nabla}_{X} Y=\nabla_{X} Y+\varepsilon H(X, Y) \xi, \quad \tilde{\nabla}_{X} \xi=-A(X)
$$

respectively, where $X, Y$ are vector fields tangent to $M, H$ is the second fundamental tensor and $A$ is the shape operator of $M$ in $N$ and $g(A(X), Y)=H(X, Y)$. Furthermore, for $k>1$, we also have that $H^{k}(X, Y)=g\left(A^{k}(X), Y\right), \operatorname{tr}\left(H^{k}\right)=\operatorname{tr}\left(A^{k}\right)$, $k \geq 1, H^{1}=H$ and $A^{1}=A$. We denote by $R$ and $\tilde{R}$ the Riemannian-Christoffel curvature tensors of $M$ and $N$, respectively.

The Gauss equation of $M$ in $N$ has the following form:

$$
R\left(X_{1}, X_{2}, X_{3}, X_{4}\right)=\tilde{R}\left(X_{1}, X_{2}, X_{3}, X_{4}\right)+\varepsilon \bar{H}\left(X_{1}, X_{2}, X_{3}, X_{4}\right) .
$$


From now on, we will assume that $M$ is a hypersurface in a semi-Euclidean space $E_{s}^{n+1}$. So, Eq.(3.1) turns into

$$
R\left(X_{1}, X_{2}, X_{3}, X_{4}\right)=\varepsilon \bar{H}\left(X_{1}, X_{2}, X_{3}, X_{4}\right),
$$

where $X_{1}, X_{2}, X_{3}, X_{4}$ are vector fields tangent to $M$ and $\bar{H}=\frac{1}{2} H \widetilde{\wedge} H$. From (3.2), by contraction, we get easily

$$
S\left(X_{1}, X_{4}\right)=\varepsilon\left(\operatorname{tr}(H) H\left(X_{1}, X_{4}\right)-H^{2}\left(X_{1}, X_{4}\right)\right) .
$$

Moreover, by contracting (3.3), we obtain

$$
\kappa=\varepsilon\left(\operatorname{tr}(H)^{2}-\operatorname{tr}\left(H^{2}\right)\right)
$$

Now we give the following Lemmas which will be used in the main results.

Lemma 3.1. [6] Let $E$ and $D$ be two symmetric $(0,2)$-tensors at point $x$ of a semi-Riemannian manifold $(M, g)$. If the condition

$$
\alpha Q(g, E)+\gamma Q(E, D)+\beta Q(g, D)=0 ; \quad \alpha, \beta, \gamma \in \mathbb{R}, \gamma \neq 0
$$

is satisfied at $x$, then the tensors $E-\frac{1}{n} \operatorname{tr}(E) g$ and $D-\frac{1}{n} \operatorname{tr}(D) g$ are linearly dependent.

Lemma 3.2. [6] Any hypersurface $M$, immersed isometrically in an $(n+1)$-dimensional semi-Euclidean space $E_{s}^{n+1}, n \geq 4$, satisfies the condition

$$
R \cdot R=Q(S, R) .
$$

Proposition 3.1. Let $M$ be a hypersurface in a semi-Euclidean space $E_{s}^{n+1}, n \geq 4$, then we have

$$
\widetilde{P} \cdot R=-(a+b) R \cdot R+\frac{\kappa}{n}\left[\frac{a}{n-1}+b\right] Q(g, R) .
$$

Proof. Let $X_{h}, X_{i}, X_{j}, X_{k}, X_{l}, X_{m} \in \chi(M)$. So using (2.3) we have

$$
\begin{aligned}
\left(\widetilde{P}\left(X_{h}, X_{i}\right) \cdot R\right)\left(X_{j}, X_{k}, X_{l}, X_{m}\right)= & -R\left(\widetilde{P}\left(X_{h}, X_{i}\right) X_{j}, X_{k}, X_{l}, X_{m}\right) \\
& -R\left(X_{j}, \widetilde{P}\left(X_{h}, X_{i}\right) X_{k}, X_{l}, X_{m}\right) \\
& -R\left(X_{j}, X_{k}, \widetilde{P}\left(X_{h}, X_{i}\right) X_{l}, X_{m}\right) \\
& -R\left(X_{j}, X_{k}, X_{l}, \widetilde{P}\left(X_{h}, X_{i}\right) X_{m}\right) .
\end{aligned}
$$

Then using (2.1), (2.4) and (2.7), we have

$$
\widetilde{P} \cdot R=a H \widetilde{\wedge} Q\left(H^{2}, H\right)-b Q(S, R)+\frac{\kappa \varepsilon}{n}\left[\frac{a}{n-1}+b\right](H \widetilde{\wedge} Q(g, H) .
$$

Thus, by (2.9), Eq. (3.8) turns into

$$
\widetilde{P} \cdot R=a Q\left(H^{2}, \bar{H}\right)-b Q(S, R)+\frac{\kappa \varepsilon}{n}\left[\frac{a}{n-1}+b\right] Q(g, \bar{H}) .
$$

By using (3.3), (3.2) and Lemma 3.2, the Eq. (3.9) can be rewritten as

$$
\widetilde{P} \cdot R=-(a+b) R \cdot R+\frac{\kappa}{n}\left[\frac{a}{n-1}+b\right] Q(g, R) .
$$

This completes the proof of the proposition.

As an immediate consequence of Proposition 3.1, we have the following theorem: 
Theorem 3.1. Let $M$ be a hypersurface in a semi-Euclidean space $E_{s}^{n+1}, n \geq 4$. If the condition $\widetilde{P} \cdot R=0$ holds on $M$, then $M$ is pseudosymmetric.

Lemma 3.3. [3] Let $M$ be a hypersurface in a semi-Euclidean space $E_{s}^{n+1}, n \geq 3$. Then $M$ is pseudosymmetric if and only if $R \cdot R=0$ or the second fundamental tensor $H$ of $M$ satisfies the condition

$$
H^{2}=\alpha H+\beta g \quad \alpha, \beta \in \mathbb{R} .
$$

Definition 3.1. Let $M$ be a hypersurface in a semi-Euclidean space $E_{s}^{n+1}, \mathrm{n} \geq 4$. If $\widetilde{P} \cdot S=0$, then $M$ is called pseudo projectively Ricci-semisymmetric.

Lemma 3.4. Let $M$ be a hypersurface in a semi-Euclidean space $E_{s}^{n+1}, n \geq 4$. If $M$ is pseudo projectively Ricci-semisymmetric, then there is a real valued function $\lambda$ on $M$ such that

$$
H^{2}=\lambda H+\frac{1}{n}\left(\operatorname{tr}\left(H^{2}\right)-\lambda \operatorname{tr}(H)\right) g .
$$

Proof. Let $X_{h}, X_{i}, X_{j}, X_{k} \in \chi(M)$. So, by using (2.3), we have

(3.12) $(\widetilde{P} \cdot H)\left(X_{h}, X_{i} ; X_{j}, X_{k}\right)=-H\left(\widetilde{P}\left(X_{j}, X_{k}\right) X_{h}, X_{i}\right)-H\left(X_{h}, \widetilde{P}\left(X_{j}, X_{k}\right) X_{i}\right)$ and, similarly,

$$
\left(\widetilde{P} \cdot H^{2}\right)\left(X_{h}, X_{i} ; X_{j}, X_{k}\right)=-H^{2}\left(\widetilde{P}\left(X_{j}, X_{k}\right) X_{h}, X_{i}\right)-H^{2}\left(X_{h}, \widetilde{P}\left(X_{j}, X_{k}\right) X_{i}\right) .
$$

Then, by making use of (2.1), (2.4) and (3.2), we get

$$
\widetilde{P} \cdot H=(a+b) \varepsilon Q\left(H, H^{2}\right)-\frac{\kappa}{n}\left[\frac{a}{n-1}+b\right] Q(g, H)
$$

and

$$
\begin{aligned}
\widetilde{P} \cdot H^{2}= & a \varepsilon Q\left(H, H^{3}\right)+b \varepsilon \operatorname{tr}(H) Q\left(H, H^{2}\right) \\
& -\frac{\kappa}{n}\left[\frac{a}{n-1}+b\right] Q\left(g, H^{2}\right) .
\end{aligned}
$$

Since $M$ is pseudo projectively Ricci-semisymmetric, by using (3.3), we have

$$
\widetilde{P} \cdot S=\varepsilon\left(\operatorname{tr}(H) \widetilde{P} \cdot H-\widetilde{P} \cdot H^{2}\right)=0 .
$$

Thus, by substituting (3.14) and (3.15) into (3.16), we obtain

$$
\begin{aligned}
& a \operatorname{tr}(H) Q\left(H, H^{2}\right)-a Q\left(H, H^{3}\right)-\frac{\varepsilon \kappa}{n}\left[\frac{a}{n-1}+b\right] \operatorname{tr}(H) Q(g, H) \\
& +\frac{\varepsilon \kappa}{n}\left[\frac{a}{n-1}+b\right] Q\left(g, H^{2}\right)=0 .
\end{aligned}
$$

Hence, from (3.17), by a contraction, we have

$$
\begin{aligned}
H^{3}= & \operatorname{tr}(H) H^{2}+\left[-\operatorname{tr}\left(H^{2}\right)+\frac{\operatorname{tr}\left(H^{3}\right)}{\operatorname{tr}(H)}-\frac{\varepsilon \kappa}{a}\left(\frac{a}{n-1}+b\right)\right] H \\
& +\frac{\varepsilon \kappa}{a \operatorname{tr}(H)}\left[\frac{a}{(n-1)}+b\right] H^{2} \\
& +\left[\left(\frac{\varepsilon \kappa \operatorname{tr}(H)}{a \cdot n}-\frac{\varepsilon \kappa \operatorname{tr}\left(H^{2}\right)}{a \cdot n \operatorname{tr}(H)}\right)\left[\frac{a}{(n-1)}+b\right]\right] g .
\end{aligned}
$$


So, by substituting (3.18) into (3.17), we get

$$
\begin{aligned}
& -\frac{\varepsilon \kappa}{\operatorname{tr}(H)}\left[\frac{a}{(n-1)}+b\right] Q\left(H, H^{2}\right)-\frac{\varepsilon \kappa \cdot \operatorname{tr}\left(H^{2}\right)}{n \cdot \operatorname{tr}(H)}\left[\frac{a}{(n-1)}+b\right] Q(g, H) \\
& +\frac{\varepsilon \kappa}{n}\left[\frac{a}{(n-1)}+b\right] Q\left(g, H^{2}\right)=0
\end{aligned}
$$

Then, by Lemma 3.1, the tensors

$$
H^{2}-\frac{1}{n} \operatorname{tr}\left(H^{2}\right) g
$$

and

$$
H-\frac{1}{n} \operatorname{tr}(H) g
$$

are linearly dependent, which proves the lemma.

Hence, by combining Lemma 3.3 and Lemma 3.4, we have the following theorem:

Theorem 3.2. Let $M$ be a hypersurface in a semi-Euclidean space $E_{s}^{n+1}, n \geq 4$. If $M$ is pseudo projectively Ricci-semisymmetric, then $M$ is pseudosymmetric.

Theorem 3.3. Let $M$ be a hypersurface in a semi-Euclidean space $E_{s}^{n+1}, n \geq 4$. If $M$ is pseudo projectively Ricci-semisymmetric, then $M$ is Ricci-pseudosymmetric.

Proof. By using (2.1), (2.3) and (2.4), we have

$$
(\widetilde{P} \cdot S)=a(R \cdot S)-\frac{\kappa}{n}\left[\frac{a}{n-1}+b\right] Q(g, S) .
$$

Since the condition $\widetilde{P} \cdot S=0$ holds on $M$, we get

$$
R \cdot S=\frac{\kappa}{n}\left[\frac{1}{n-1}+\frac{b}{a}\right] Q(g, S) .
$$

This completes the proof of the theorem.

Lemma 3.5. [2] Let $M$ be a hypersurface in a semi-Euclidean space $E_{s}^{n+1}, n \geq 4$. $M$ satisfies the condition

$$
R \cdot S=Q\left(H, \operatorname{tr}(H) H^{2}-H^{3}\right) .
$$

Theorem 3.4. Let $M$ be a hypersurface in a semi-Euclidean space $E_{s}^{n+1}, n \geq 4$. If $M$ the condition $H^{2}=\operatorname{tr}(H) H$ holds on $M$, then $M$ is pseudo projectively Riccisemisymmetric.

Proof. Since $H^{2}=\operatorname{tr}(H) H$ and $H^{k}(X, Y)=g\left(A^{k}(X), Y\right)$, we have

$$
H^{3}=\operatorname{tr}(H) H^{2} \text {. }
$$

So, by substituting (3.22) into (3.21), we get $R \cdot S=0$. Thus, Eq.(3.20) turns into

$$
(\widetilde{P} \cdot S)=-\frac{\kappa}{n}\left[\frac{a}{n-1}+b\right] Q(g, S) .
$$

Since $H^{2}=\operatorname{tr}(H) H$, by using (3.22) and (3.3), we get $Q(g, S)=0$. In the proof of this theorem which proves that $M$ is pseudo projectively Ricci-semisymmetric. 
Example 3.1. Let $\mathbf{S}^{2}=\left\{p \in \mathbb{R}^{3}\right.$ such that $\left.|p|=1\right\}$ be the standard unit sphere. First we consider

$$
M^{4}=\mathbf{S}_{1}^{2} \times \mathbf{S}_{2}^{2}=\left\{(p, q) \in \mathbb{R}^{6}=\mathbb{R}^{3} \times \mathbb{R}^{3} \text { such that }|p|=|q|=1\right\} .
$$

Next we take the cone

$$
\mathbf{C}^{5}=\left\{(t p, t q) \in \mathbb{R}^{6} \text { such that }|p|=|q|=1, \quad t>0, \mathrm{t} \in \mathbb{R}\right\} .
$$

In [1], the authors show that the principal curvatures of $\mathbf{C}^{5}$ are $\left(0, \frac{1}{\sqrt{2} t}, \frac{1}{\sqrt{2} t},-\frac{1}{\sqrt{2} t},-\frac{1}{\sqrt{2} t}\right)$ and the cone $\mathbf{C}^{5}$ is Ricci-semisymmetric, but not semi-symmetric. It can be easily seen that the cone $\mathbf{C}^{5}$ satisfies the condition $\widetilde{P} \cdot S=0$ and it is pseudosymmetric.

\section{REFERENCES}

[1] Abdalla B.E., Dillen F.A., A Ricci-semi-symmetric hypersurface of Euclidean space which is not semi-symmetric. Proc Amer Math Soc. 130, 1805.(2002).

[2] Defever F., Deszcz R., Dhooghe, P., Verstraelen L., Yaprak Ş., On Ricci-pseudosymmetric hypersurfaces in spaces of constant curvature. Results in Math. 27, 227-236 (1995).

[3] Deszcz R., On certain classes of hypersurfaces in space of constant curvature. Geometry and Topology of Submanifolds VIII, World .Sci., Singapore. 101-110 (1996).

[4] Deszcz R.,Glogowska M., Some nonsemisymmetric Ricci-semisymmetric warped product hypersurfaces. Pulb Inst Math Beograd (N.S.). 72, 81-93.(2002).

[5] Deszcz R., On pseudosymmetric spaces. Bull Soc Belg Math Ser A. 44, 1-34.(1992).

[6] Deszcz R., Verstraelen L., Yaprak Ş., Hypersurfaces with pseudosymmetric Weyl tensor in conformally flat manifolds. Geometry and Topology of Submanifolds IX. Valenciennes/Lyon/Leuven: World Scientific Publishing. 131-77 (1997).

[7] Dabrowska M., Defever F., Deszcz R. and Kowalczyk D., Semisymmetry and Riccisemisymmetry for hypersurfaces semi-Euclidean spaces. Publ. Inst. Math. (Beograd) (N.S.) 67, 103-111 (2000).

[8] Özgür C., Hypersurfaces satisfying some curvature conditions in the semi-Euclidean space. Chaos, Solitons and Fractals. 39, 2457-2464.(2009).

[9] Özgür, C., Arslan, K., On some class of hypersurfaces in $\mathrm{E}^{n+1}$ satisfying Chen's equality. Turkish J. Math. 26, 283-293 (2002).

[10] Özgür, C., Arslan, K., Murathan, C., Conharmonically semi-parallel hypersurfaces. Proceedings of the Centennial "G. Vranceanu", Part II (Bucharest, 2000). An. Univ. Bucureşti Mat. Inform. 50, 121-132 (2001).

[11] Prasad B., A pseudo projective curvature tensor on a Riemannian manifold, Bull. Calcutta Math. Soc. 94, 163-166 (2002).

[12] Szabo ZI., Structure theorems on Riemannian spaces satisfying $R(X, Y) \cdot R=0$. I. local version J. Diff. Geom. 17, 531-82 (1982). 